\title{
Akupunktur: hauptsächlich Placebo?
}

\section{Ist die Schmerzreduktion unter Akupunktur nur Placebo oder mehr?}

- Wiener Gynäkologen randomisierten 40 Patientinnen, die sich einer Laparoskopie unterziehen mussten, in zwei Gruppen. Diese erhielten entweder Ohrakupunktur mit Elektrostimulation oder eine Scheinakupunktur.

Der Hauptzielparameter der Studie war der postoperative Schmerz. Die Nachbeobachtung erfolgte bis 72 Stunden nach dem Eingriff. Im Gruppenvergleich zeigte sich kein Effekt der Akupunktur auf die Schmerzen oder auf den Schmerzmittelbedarf. Die Autoren meinen daher, dass die analgetischen Effekte der Akupunktur „hauptsächlich auf einem Placeboeffekt beruhen“.

\section{Kommentar}

Dies ist eine elegante und bedeutsame Studie. Experten sind sich nach wie vor uneinig darüber, ob die Schmerzreduktion nach Akupunktur mehr als ein Placeboeffekt ist. Da Akupunktur immer einen Therapeuten-Patient-Kontakt voraussetzt, kann es schwierig sein, spezifische von unspezifischen Therapieeffekten zu trennen. Einige Untersuchungen zeigen z. B., dass die zwischenmenschlichen Interaktionen hier von entscheidender Bedeutung sind. In der vorliegenden Untersuchung wurden die Akupunkturnadeln gesetzt, als die Patienten unter Vollnarkose standen. Zwischenmenschliche Interaktionen oder Plazeboeffekte sind daher so gut wie ausgeschlossen. Die Tatsache, dass unter diesen Bedingungen keine analgetischen Effekte verifizierbar waren, spricht also sehr stark dafür, dass Akupunktur in der Schmerztherapie keine spezifischen Effekte induziert.

\section{- A. Holzer et al.}

Auricular acupuncture for postoperative pain after gynecological surgery: a randomized clinical trial. Minerva Anastesiologica 77 (2011) 298-304 